\title{
El estrés laboral y sus incidencias en los trabajadores del sector salud. Estudio de caso Unidad de Conductas Adictivas Psico
}

\section{Labor stress and its effect in healthcare workers. Case study in an "Addictive conduct unit Psico" in Cartago, Valle del Cauca, Colombia.}

Eliana Amparo Rico Arango', Guillermo Antonio Herrera Rojas', Yovany Ospina Nieto²

' Universidad Pontificia Bolivariana-Seccional Palmira.

${ }^{2}$ Grupo de Investigación en Pedagogía y Desarrollo Humano. Coordinación de Investigación Universidad Pontificia Bolivariana, sede Palmira, Valle del Cauca-Colombia. Autor para correspondencia: yovany.ospina@upb.edu.co

\section{Resumen}

En este artículo se compendia la metodología, la base teórica y los hallazgos del estudio de caso realizado en la Unidad de Conductas Adictivas -Psico, cuyo objetivo era evaluar la incidencia del estrés laboral entre sus empleados. Para lograrlo, se diseñó un proceso investigativo documental que consideró diferentes escritos; abordando aspectos, tales como clima organizacional, talento humano y competencias laborales. Al final, se cualificó el nivel de estrés laboral y se formuló un plan de acción tendiente a disminuir las causas estresoras. En la Metodología, se utilizó el modelo de investigación cualitativa, pues se trataba de un fenómeno social: el rol laboral. Fue un estudio transversal acerca de indicadores de estrés para profesionales y tecnólogos de la salud, que se aplicó a los colaboradores de la empresa Psico (Unidad de Conductas Adictivas), ubicada en Cartago, Valle del Cauca. El test incluyó todos los niveles organizacionales de la institución: jefes, profesionales, auxiliares y operarios.

Palabras clave: estrés, incidencia, psico, salud, trabajadores.

\section{Abstract}

In this article, it condenses the methodology, the theory base and the findings of the study case, realized in the Unit of Addictives Behaviors - Psycho, its objective was evaluate the incidence 
of labor stress among its employees. To achieve, it was designed a documentary investigative process that considered different writtens; abording aspects, such as organizational climate, human talent and labor competencies. At the end, it was qualified the level of labor stress and it was formulated an action plan tending to decrease the stressor causes. In the Methodology, it was used the qualitative research model, being as it was about of a social phenomenon: the labor role. It was a transversal study about stress indicators for professionals and health technologists, which applied to the collaborators of the Pshyco company (Unit of Addictives Behaviors), it located in Cartago - Valley. The test included all of organizational levels of the institution: chiefs, professionals, auxiliaries and operatives.

Keywords: stress, advocacy, psycho, health, workers.

\section{Introducción}

El estrés es un padecimiento que puede desencadenar daños psicológicos y fisiológicos en las personas que lo padecen; en ocasiones, pueden ser permanentes, pues limitan el desempeño laboral.

Hoy en día se reconoce que el estrés laboral es uno de los principales problemas para la salud de los trabajadores y del buen funcionamiento de las entidades para las que trabajan" "Es una enfermedad peligrosa para las economías industrializadas y en vías de desarrollo, perjudicando a la producción, al afectar a la salud física y mental de los trabajadores (Organización Internacional del Trabajo (OIT), 1986-1992).

El estrés laboral se manifiesta cuando se hace evidente un desacuerdo entre el individuo, su lugar de trabajo y la organización. En tal perspectiva, se entiende como la manera de responder del individuo, ante las demandas excesivas o amenazantes de su medio laboral, y que pueden poner en riesgo su bienestar o integridad. Se ha señalado que el estrés es un factor importante para considerar, por su incidencia en la salud profesional; hoy por hoy, una fuente significativa de incapacidad laboral.

Esta serie de consideraciones permite establecer el punto de partida para elaborar un plan de acción que logre disminuir los efectos estresores, en los empleados de la Unidad de Conductas Adictivas Psico. Dicho plan estará acompañado de una herramienta de diagnóstico y análisis. Se trabajará con las relaciones, las ideas y las experiencias personales de los trabajadores; con sus factores genéticos, las influencias ambientales y las exigencias y condiciones que se manifiestan, contrarias a las necesidades y aspiraciones personales del medio ambiente de trabajo.

Se sabe que los factores de riesgo psicosocial tienen manifestaciones en patologías orgánicas, con mecanismos de diversos tipos: cognoscitivos, afectivos, conductuales, fisiológicos, psicosomáticas-emocionales y alteraciones del comportamiento. Dichos factores aparecen asociados, en el ámbito laboral, con la presencia de tareas ambiguas, turnos rotativos, ausencia de pausas activas, tareas repetitivas, inseguridad del empleo, falta de asertividad directiva, incrementos injustificados de la carga laboral. Este último factor se intensifica con una inadecuada remuneración salarial.

La Organización Internacional del trabajo (OIT) y la OMS (1984) consideran la clasificación de estos riesgos en tres categorías básicas: a) Del individuo, b) Del medio laboral, c) Del entorno extra laboral. En este marco de ideas, cobra, entonces, relevancia la pregunta que constituye la tesis central de este abordaje argumentativo: ¿Cuál es la influencia que tiene el estrés laboral en el desempeño de los trabajadores de la Unidad de Conductas Adictivas Psico?

Por lo anterior, es necesario identificar los grados de incidencia de los factores estresores en el personal de la institución. Ello permitirá no solo reconocer su presencia, sino que posibilitará la construcción de acciones de intervención. Con las cuales, se podrá consolidar un plan de trabajo encaminado a reducir los efectos negativos que dichos factores poseen en el desempeño laboral y en la calidad de vida de los colaboradores de Psico. Para desarrollar esta labor, se diseñó una encuesta, en la que se incluyen elementos que -adicionalmente a la identificación de los agentes estresores- coadyuvan al proceso de cuantificación de los niveles de incidencia de dichos agentes. Esta es una condición necesaria para lograr la construcción de las acciones de intervención mencionadas. 
La aplicación de la encuesta es un factor fundamental para el análisis del ambiente laboral, puesto que solo, mediante la implementación de procesos de investigación en la fuente, es posible la obtención de información fidedigna acerca de los agentes estresores específicos del entorno laboral, objeto del presente abordaje. Dicha información, una vez obtenida y analizada, será el insumo que dará paso a la construcción e implementación de un plan de trabajo encaminado a la reducción de los efectos adversos del estrés, tanto en la esfera laboral como social y humana de los colaboradores de Psico. Incluso, puede servir para el abordaje de la problemática en sectores relacionados.

Una vez planteado el escenario, se empieza a reconocer el entorno del trabajador y sus implicaciones, puesto que son la base y el fundamento para el modelo de vida. De hecho, es donde el hombre establece sus relaciones con la naturaleza, buscando la plena satisfacción de sus necesidades y aspiraciones; así es como logra desarrollarse tanto en lo individual como en lo social. Tal relación da origen al medio laboral, en el que el trabajador interactúa con diferentes condiciones o problemáticas que bien pueden impactar su vida de modo positivo o negativo.

Se establece, entonces, una relación directa entre la salud y el mundo de lo laboral, entendida como el vínculo que se origina entre individuo (trabajador) y la tarea que ejecuta y las influencias que sobre la salud implican dichas labores. Este conjunto de variables que definen la realización de la tarea y el entorno en que esta se realiza se denominan condiciones de trabajo y están constituidas por factores del ambiente, de la tarea o labor y de la estructura de la organización para la que esta se ejecuta.

Los factores de riesgo psicosocial son la interacción de las relaciones entre las variables que caracterizan al trabajador, las condiciones particulares de la tarea y el entorno que lo rodea; lo cual, en determinadas circunstancias, pueden afectar de modo negativo tanto a la estructura organizativa como al trabajador. Por este motivo, se requiere una aproximación más puntual en material de riesgos psicosociales; en especial, en aquellos sectores de profesionales, como los trabajadores de la Unidad de Conductas Adictivas Psico, quienes por su labor, enfrentan una multiplicidad de factores de riesgo, los cuales deben ser abordados en su estudio, de un modo más específico.

\section{Desarrollo del caso}

Como ya se dijo, el estrés es un padecimiento que puede desencadenar daños psicológicos y fisiológicos en las personas que lo padecen; en ocasiones, pueden ser permanentes, pues limitan el desempeño laboral. Efectivamente, en los últimos años, se han incrementado las alarmas sobre la pérdida de la productividad y la eficiencia generada por el estrés laboral. Cuando el estrés se transforma en cotidianidad, son comunes síntomas, como: ansiedad, inquietud, nerviosismo, temor o angustia. La dificultad de ello estriba en que es casi imposible darnos cuenta de tal problemática, si no se cuenta con una reflexión consciente de la situación (Peiró, 2000).

De igual manera, es importante observar las actitudes comportamentales que los empleados desarrollan hacia los usuarios de la organización. Es decir, hacia el servicio que ofrecen, puesto que está asociado a los riesgos laborales de su entorno de trabajo. Los riesgos de orden psicosocial son de especial relevancia, puesto que el equipo humano trabaja en continua interacción con personas.

En dicho entorno, es pertinente definir el término "colaborador", utilizado por Vecino (2011) para referirse al trabajador o empleado. Esta conceptualización busca brindar una proyección del individuo en condiciones de integralidad, pues se reconoce no solo la utilización de sus capacidades físicas y mentales, sino también lo actitudinal; es decir, una concepción holística del ser. En la opinión pública, queda muy bien referirse a los trabajadores como "colaboradores"; aunque enmascara otra realidad.

Desde los años 70, la Administración de Empresas ha desarrollado diversos discursos y teorías para explicar los cambios en la organización de los procesos productivos. La base de estos cambios opera en el paso del modelo fordista a algo llamado posfordismo. Se denomina fordismo a una manera específica de organizar la producción, cuya base es la planta-fábrica de la empresa automotriz Ford. Son fábricas de muchos obreros, organizadas en líneas de producción, con actividades mecanizadas y una tendencia a automatizarlo todo. El fordismo es el modelo de la gran producción, en la gran fábrica. Es difícil hablar de fordismo, en sentido estricto, por 
el tamaño de nuestras unidades de producción y la ausencia de una real industrialización (Mejía, 2014).

Todo ello dio pie a plantear procesos de"flexibilidad laboral" (Mejía, 2014). Se trata de unos modelos de contratación que hacen factible una mayor rotación de empleos y, por tanto, generan inestabilidad laboral. Hoy día, para que un individuo pueda mantener su puesto de trabajo, ya no le basta una profesión, debe tener competencia en el desarrollo de múltiples habilidades.

El impacto de este nuevo planteamiento en las relaciones laborales es muy fuerte. El individuo es fácilmente sustituible. En contravía de lo que podría pensarse a partir de las nuevas concepciones del mundo del trabajo y las nuevas tecnologías, que si bien han supuesto un nuevo rol para el trabajador, no han significado una mejora real de sus necesidades individuales y colectivas. Todos estos factores implican una inmensa carga psicosocial que genera entornos laborables no saludables y, por consiguiente, se constituyen en factores generadores de estrés.

\section{Estrategias empresariales}

El aspecto organizacional moderno comporta rasgos positivos, por medio de los cuales las organizaciones han redescubierto la valía de los 'colaboradores', como recursos humanos. Esto lo ha hecho, en buena medida, a partir del trabajo de Becker (1964). Además, han resignificado la experiencia que brinda el desarrollo continuado de un oficio; así, le han dado un mayor valor al ser humano. Cabe señalar, entonces, que el descubrimiento organizacional más significativo estriba en la concepción más eficiente de la gestión de los recursos humanos, puesto que los considera más como personas, tal como lo enfatiza Casado (2003). Al entender el talento humano, como personas activas y agentes de su propio accionar, se facilita la gestión estratégica (Bonache \& Cabrera, 2002). Ello comporta la necesidad de recuperar el respeto, como una norma básica en el desarrollo de las relaciones laborales (Sennet, 2003).

Tal como lo ha señalado Senge (1997), el replanteamiento de esta perspectiva, o su revival, ha dado lugar a estrategias empresariales que definen la organización como un sistema de producción e intercambio de experiencia y conocimientos; esta tesis es apoyada por Goleman \& Cherniss (2005), quienes enfatizan que se ha otorgado, por demás, la resignificación del componente emocional, como un límite adicional para funcionamiento empresarial, con todos sus matices. De esta manera, se entendió, inicialmente, el recurso humano como una extensión utilitarista: se asumió, de modo real, como capital intelectual; y después, como capital psicológico, según Luthans \&Youssef (2007).

Aun, si se toma como punto de vista una concepción, desde lo funcional -como lo es la productividad- "las organizaciones laborales han hecho un largo recorrido desde el primer industrialismo y las formulaciones tayloristas y fordistas" (Moreno-Jiménez, Garrosa, González, 2000); por cierto, esta influencia va hasta las concepciones modernas. Cabe destacar, en este contexto, los desarrollos en los conceptos de calidad de vida, responsabilidad social corporativa y la acción social de las empresas.

De igual manera, estos últimos autores argumentan que -respecto a la calidad de vida laboral- es importante resaltar que esta no puede ser evaluada exclusivamente por elementos subjetivos y emocionales, como la satisfacción laboral, sino que es necesaria la búsqueda de marcadores objetivos. En un marco general, tales apreciaciones han permitido dar paso al concepto de organizaciones saludables; así lo expresan Henry (2005, p. 383-392) y Cooper \& Williams (1994): "las organizaciones que cumplen sus objetivos comerciales y económicos y facilitan el desarrollo profesional y personal de sus trabajadores".

A la par de estas virtudes, los puntos negros no son pocos. La álgida dinámica en que se desenvuelven las empresas presiona diversos cambios que incluyen sectores organizacionales, tecnológicos, comerciales y económicos. La aceleración de la historia causa desequilibrios y produce nuevos riesgos; precisamente por ello, nuestra sociedad actual ha sido conceptualizada como "La sociedad del riesgo" (Beck, 2006) y (Beck, 2008), una sociedad dominada por relaciones y situaciones sobre las que se tiene escaso control, lo que lleva a una generalización de la inseguridad, del miedo y del estrés.

En tal marco de ideas, han surgido nuevos riesgos de toda índole. Especialmente, la preocupación en el 
marco laboral de los llamados "riesgos emergentes" (OIT, 2010), provenientes de campos tan diversos, como las nuevas tecnologías, los nuevos sistemas de producción, las nuevas materias primas y los nuevos compuestos químicos y biológicos. El Estudio de la Agencia Europea para la Seguridad y la Salud en el Trabajo, sobre los "riesgos psicosociales emergentes" (OIT, 2010), estableció una lista de 42 riesgos psicosociales; gran parte de los cuales están causados por un doble fenómeno: la expansión del sector terciario de la producción, el sector de servicios y el proceso de la globalización en sus múltiples facetas; una de las cuales es la del mercado de trabajo. Ambos procesos están estrechamente asociados.

El sector servicios del mundo laboral ha crecido exponencialmente desde la mitad del siglo XX. Bell (1994) -en su obra de referencia básica, en Ciencias Sociales, El advenimiento de la sociedad Posindustrial- especifica que la economía actual se ha desplazado de los bienes a los servicios, y ha creado un nuevo y expansivo mercado con nuevas condiciones de trabajo.

Los riesgos laborales del mercado de servicios son, principalmente, psicosociales, provenientes de las nuevas formas de trabajo, del dinamismo de las nuevas organizaciones y sus formas cambiantes. Son riesgos derivados de las múltiples formas de atender a una clientela, en la interacción dinámica con el trabajador. El trabajo emocional ilustra este nuevo tipo de riesgos psicosociales. Un buen número de trabajos asistenciales y de servicios exigen, hoy día, la autorregulación de las emociones, es decir, la inhibición de las emociones negativas y la expresión forzada de emociones positivas. En un caso y otro, los efectos son de desgaste emocional, de disonancia emocional. Si la necesidad de asegurar la satisfacción del cliente es grande, la exigencia del trabajo emocional es muy importante (Hochschild, 1983). No se pide solo un servicio, sino uno emocionalmente reforzante para el cliente. El agotamiento resultante de esta constante actitud interna y externa, puede ser extenuante y notoriamente contraproducente para los indicadores de la competitividad.

Las estrategias organizacionales -empleadas con miras a lograr y mantener la competitividad, pero no su hegemonía- han ido desde la deslocalización de las empresas hacia mercados legalmente más desregulados, o la utilización de estrategias de subcontra- tación o de outsourcing que facilitaba una disminución del coste total" (Castillo, 2008). Probablemente, la operación estratégica más habitual ha consistido en la adquisición y fusión de las empresas, a fin de obtener el dominio del mercado, la supresión de la competencia, el control de los sistemas de producción y la capacidad de imponer el precio final de los productos (Zozaya, 2007). "Otro sistema habitual ha consistido en la disminución de plantillas (downsizing) para aumentar beneficios y reducir costes" (Jiménez \& Sánchez 2005). Las consecuencias de todas estas estrategias han sido la aparición de nuevos riesgos emergentes organizacionales, la acumulación del riesgo global y el aumento de la siniestralidad (Benavides, Delclos, Benach \& Serra, 2006).

La situación planteada no ha variado mucho y su vigencia parece aun mayor ante la continuidad de la problemática relacionada con la inseguridad en los aspectos de lo contractual. Las grandes empresas acordaron muchas de las políticas mundiales, a lo largo de la historia contemporánea; esta determinación ha pasado a manos de los mercados económicos globales, específicamente, a la de los sectores del mundo financiero. Como una consecuencia inmediata y global, ha habido un hundimiento del mercado del trabajo y aumento de la inseguridad laboral en todas sus formas; principalmente, de la contractual (Becker, 2000). La ausencia de una estabilidad laboral se constituye, así, como el más preponderante de los riesgos psicosociales en el actual mundo del trabajo.

Los procesos de industrialización -especialmente, los relacionados con la automatización de la producción- han dado lugar a la reducción de los espacios de trabajo y a la disminución consecuente de la cantidad de mano de obra empleada; esto es, a la flexibilidad laboral, descrita por el aumento de los horarios. Todo esto implica: a) una supervisión más completa y permanente y b) la necesidad de un clima laboral estable, es decir, al logro de la "paz laboral" (Mejía 2014). Este concepto es concordante con los planteamientos de las organizaciones saludables, planteado por Henry (2005, p. 383-392) y Cooper \& Williams (1994).

Si bien, a lo largo de la historia del mundo laboral, se ha contado con mecanismos y figuras que buscan preservar la denominada 'paz laboral', la percepción del concepto, su evolución y funciones han variado en 
la medida en que se ha ido creando conciencia acerca de que las personas cumplen un papel vital para el desarrollo de las organizaciones. Aunque tal valor siempre es dimensionado en relación con su capacidad de generar valor agregado, en virtud del conocimiento que reside en ellas. La selección de una persona, para el desarrollo de una determinada labor, se basa en la escogencia del candidato que más logre aproximarse a los perfiles creados por la organización, los cuales, indistintamente, privilegian los posibles resultados del seleccionado, en términos de productividad. Debe quedar claro que se busca no necesariamente una persona que desarrolle sentido de pertenencia con la organización, sino que, más bien, aporte su conocimiento, desarrolle nuevo conocimiento y los aplique, los comparta y, en buena medida, engrose los activos para futuros procesos para desarrollar.

Las organizaciones han entendido la importancia de generar sentido de pertenencia en sus colaboradores, y de lograr el máximo grado de desarrollo de sus potencialidades. De hecho, conocen la efectividad de las posibilidades de uso y reúso en estos desarrollos y en la búsqueda de un verdadero desarrollo organizacional. De este modo, es posible entender por qué se habla de Capital Humano con mayor propiedad, más que de Talento Humano.

En la construcción del Capital Humano, es fundamental contar con una hoja de ruta clara y precisa, una planeación estratégica. Con ella, es posible crear un clima laboral saludable; por ende, la consolidación de procesos que posibiliten una cultura organizacional adecuada. Cuando estos elementos claves están establecidos, se advierte la necesidad de contar con procesos de Gestión del Capital Humano, correctamente estructurados e informados. Una vez se ha logrado avanzar, se llega a otro aspecto clave: la apropiación y uso de medios tecnológicos que apoyen el proceso. Seguidamente, se requiere la implementación de instrumentos de medición, como medio de evaluación de los indicadores de gestión. Finalmente, hay que contar con un sistema que permita la retroalimentación del proceso para llevar a cabo la toma de decisiones futuras que alineen o reorienten la planeación estratégica: basándose en los anteriores resultados y reutilización de la información.

Todo ello nos permite concluir, de manera clara, que, sin obviar su vital importancia, cuando se hable de
Talento Humano, se debe tener en cuenta su diferencia con el concepto de Capital Humano. Puesto que el primero conlleva capacidades y habilidades del individuo frente al desarrollo de una labor determinada. Y el segundo infiere la generación de valor derivada de los conocimientos, en sí, que ejecutan los 'colaboradores' y cómo estos son utilizados tanto por las personas como por la organización.

Los nuevos conceptos de la estructura organizacional, postulados a partir de los cambios propiciados por posfordismo, evidencian la necesidad de una cultura organizativa, en la que se requiere la concepción de las empresas como una familia, en la que todos los componentes actúan en favor de un bien común. No obstante, esto nos enfrenta, irremediablemente, a una realidad en la cual la relación básica del mundo laboral no ha surtido un cambio profundo, pues, en lo fundamental, subyace la misma estructura: patrón y obrero, en la que patrones y trabajadores son clases sociales diferentes.

En el tiempo transcurrido entre los años 50 y 70, 'la paz laboral' se pudo lograr, mediante acuerdos viables que garantizaban cierta estabilidad laboral y, en buena medida, una retribución económica, más o menos adecuada. Ahora, ante la dinámica globalizada y, en particular, desde la crisis del petróleo (en los 70) y las grandes transformaciones neoliberales que recortaron la función social de los Estados, el panorama es totalmente distinto. Los empresarios, preocupados más por los niveles de productividad, recurren a incentivos y estímulos simbólicos. Los aumentos salariales de antaño han cedido terreno ante reconocimientos. La negociación entre patrones y sindicatos se traduce en pequeños incentivos, como paseos de integración con rifas y obsequios, en temporadas especiales. A los tradicionales procesos de reivindicación por derechos y condiciones justas de trabajo, se les ha suplantado con mecanismos de participación para la planificación conjunta de la estrategia organizativa.

Aunque parece un hecho meramente semántico, el cambio del lenguaje es un elemento importante para definir una realidad diferente. La evolución lingüística de los términos, así como su significado, ha permitido que la palabra sea, por esencia, la manifestación del pensamiento. Esto aplica para muchas palabras que, a través del tiempo, han ido ganando en conte- 
nido, o, por lo menos, son un referente de la época que marcaron la historia del hombre" (Vecino, 2011).

Por tales razones, los trabajadores no deben ni pueden olvidar dicha condición. Los obreros, empleados, asalariados o proletarios son una clase social distinta, diferente y opuesta a los empresarios, empleadores, patrones o burgueses. Ellos tienen que "reconocer que la evolución semántica, sobre la manera de designar a la persona que labora en una organización, no necesariamente significa un cambio en la actitud o manera de relacionarse con el mismo." (Vecino, 2011).

Al reconocer la organización y la ordenación del trabajo, como condiciones de trabajo susceptibles de producir "riesgos emergentes", se da la necesidad de diagnosticar y prevenir prioritariamente los psicosociales, con el objetivo minimizar su presencia e impacto, y ofrecer entornos laborales más saludables. Dentro de los riesgos laborales de carácter psicosocial, el estrés laboral ocupa un lugar destacado, pues -tal como se ha señalado- es una de las principales causas del deterioro de las condiciones de trabajo, y fuente de ausentismo.

Gil-Monte \& Peiró (1997); Gil-Monte, Peiró \& Valcárcel (1998) han señalado que esta respuesta es resultado de la ausencia o fallo de estrategias de afrontamiento, por parte de los empleados del sector salud. Ello genera deterioro tanto en la autoestima como en las relaciones con los pacientes. Esta situación, de baja realización personal, se traslada al ambiente de trabajo y produce un agotamiento emocional. Todo este panorama desemboca en el desarrollo de un cuadro negativo de actitudes y conductas de despersonalización, como forma de afrontamiento. Además, propicia naturalmente, conductas nocivas para el individuo, somatizadas como enfermedades y alteraciones psicosomáticas (alteraciones cardiorrespiratorias, jaquecas, gastritis y úlcera, dificultad para dormir, mareos y vértigos, etc.). Que repercuten, consecuentemente, en la estructura organizativa (accidentes, deterioro del rendimiento y de la calidad asistencial o de servicios, absentismo, rotación no deseada, abandono, etc.).

El enfoque psicosocial aborda ese fenómeno, por medio del síndrome de baja autoestima personal y profesional: agotamiento físico y emocional, que se traduce en la despersonalización del individuo. Esta despersonalización es el desarrollo de sentimientos y de imagen negativa propia y de actitudes, y conductas hostiles hacia los receptores de su trabajo. Un factor de capital relevancia respecto a los resultados negativos del estrés, lo constituye, sin duda alguna, el absentismo. Este factor tiene particular incidencia en los trabajadores del área de la salud, para quienes, los costos relacionados con el estrés -depresión y ansiedad- se han incrementado en los últimos años; así, lo demuestra el Self reported Work related Illness 2006/2007(SWI), del Departamento de Seguridad y Salud del Reino Unido (HSE). Este informe señala que -para el periodo 2006/2007- se perdieron 6.756 días de trabajo, por incapacidad médica de los hombres y 7.003 días, en las mujeres. El promedio indica que los días perdidos por género fueron 34, por los hombres y 27,3, por las mujeres; lo que implica un costo diario para la empresa de US\$221.00, por los hombres y US\$319.00, por las mujeres (Grazziano \& Ferraz, 2010).

El carácter asistencial del enfermero -impregnado de su valor humanístico y unido a la diferencia entre aspiraciones vs realidad de ambiente laboral- se convierte en factor preponderante para el surgimiento del estrés y del síndrome de Burnout (Carlotto, 2005). Bianchi (1990) afirma que la naturaleza del trabajo hospitalario, aunado a la atención de múltiples personas en medio del continuo conflicto vida/muerte, se conjuga con las dificultades económicas y sociales de la categoría y de la sociedad; y lleva, a estos profesionales, a un mayor desarrollo del síndrome de burnout. De hecho, se manifiesta como un tipo específico de mecanismo de defensa y autoprotección, frente al estrés generado por la relación profesión - empleo.

El estrés del sujeto que labora debe entenderse como una respuesta a fuentes estresoras. En la Unidad de Conductas Adictivas Psico, son estresores -especialmente relevantes para el desarrollo del síndrome- las relaciones sociales de los profesionales de la salud con los pacientes y sus familiares. El estrés de estos empleados se debe a una combinación de variables físicas, psicológicas y sociales. En los que inciden, especialmente, estresores como la escasez de personal (por tanto, sobrecarga laboral), trabajo en turnos, trato con usuarios problemáticos, contacto directo con la enfermedad, el dolor $y$, en ocasiones extremas, la muerte, la falta de especificidad de funciones y tareas. 
Todo esto supone conflicto y ambigüedad de rol, falta de autonomía y autoridad en el trabajo, para poder tomar decisiones, cambios tecnológicos rápidos y bruscos, etc.

Es importante, entonces, diseñar un conjunto de estrategias para afrontar el estrés laboral. Y puede hacerse, mediante técnicas y programas, como el entrenamiento para solucionar problemas, para lograr la asertividad y para el manejo eficaz del tiempo. En el abordaje de la problemática, la intervención puede ser grupal e interpersonal, por medio de estrategias que fomenten el apoyo tanto de compañeros como de los responsables del área administrativa. En efecto, es una conjunción de acciones para brindar no solo apoyo emocional. Se requiere de un proceso de acompañamiento con evaluación periódica de los profesionales, del personal administrativo y un proceso de retroinformación sobre el desarrollo y la evolución del papel del individuo, en el proceso de la organización.

En efecto, en el desarrollo del proceso de dirección estratégica de la organización, se debe incluir el desarrollarlo de programas de prevención encaminados al mejoramiento del clima organizacional. Parte de la estrategia consistirá en desarrollar procesos previos de socialización, que propicien el acercamiento de los nuevos profesionales a la realidad laboral. De este modo, se previenen las dificultades resultantes de un desencanto, ante la ausencia de expectativas creadas. En ello, juega papel importante el desarrollo de procesos de retroalimentación, en torno a los verdaderos aspectos del ejercicio del rol como profesional de la salud.

Junto a los procesos de retroinformación grupal e interpersonal, por parte de los compañeros, se debe dar retroinformación desde la dirección de la organización y desde la unidad o el servicio, en el que se ubica el empleado. Además, es conveniente implementar programas de desarrollo organizacional.

\section{Obrero, trabajador o colaborador, más allá de lo semántico}

En términos de un estudio semiológico, la evolución lingüística de los conceptos, así como sus procesos de significación, permiten considerar las palabras como expresiones del pensamiento. Esto es aplicable a múltiples vocablos que, con el transcurrir de las épocas, han ido ganando en contenido, o, por lo menos, son un referente de épocas esenciales de la historia humana (Vecino, 2011).

La teoría organizacional está, obviamente, marcada por tales procesos de evolución. Con el advenimiento y posterior desarrollo de la revolución industrial, aparece el término obrero, derivado del concepto de clase obrera de Engels (1972). En español, comenzó a utilizarse, durante la segunda mitad del siglo XIX, como traducción de la expresión inglesa "working class" (clase trabajadora).

Los avances tecnológicos y la diversificación de la productividad organizacional traen consigo un nuevo modelo productivo. Uno, centrado en la manufactura y otro, en el que se privilegian las estructuras mentales y sus aportes, sin descuidar los procesos manufactureros. Este es el inicio de la teoría organizacional, del concepto de mano de obra calificada, que hace alusión al conocimiento que cada día le permite a la empresa ser más competitiva. En este sentido, el término obrero -entendido como"trabajador manual retribuido" - (DRAE, 2001) comienza a ser desplazado por el de trabajador que significa, en la DRAE (2001), "esfuerzo humano aplicado a la producción de riqueza". Así, se incorpora el reconocimiento del aspecto humanista en el mundo del trabajo.

A la par de este nuevo status del individuo en el medio laboral, se hace visible un cambio en el modo de designar y abordar el direccionamiento organizacional. El término para designar las áreas de dirección de las personas, en el ámbito del trabajo, presenta transformaciones propias de la evolución de dicho entorno:'relaciones industriales','recursos humanos', 'gestión humana', 'talento humano', 'gestión del capital humano'. Este tema requiere un abordaje específico: reconociendo la evolución semántica del término con que se designa al individuo, quien desarrolla una o más actividades en una organización, aun cuando dicha transformación no necesariamente conlleve una redefinición en la actitud o manera de relacionarse con la misma.

En el ámbito laboral actual, el término que más ha prevalecido, para designar a los individuos que pres- 
tan un servicio remunerado, es el de empleado.Y parece corresponder adecuadamente a la descripción de la actividad en cuestión: "ocupar a alguien, encargándole un negocio, comisión o puesto" (DRAE, 2001). No obstante, carga sobre sí una serie de interpretaciones contradictorias; quizá, por ello, el nuevo léxico organizacional plantea la exclusión del término, adoptado por el colectivo social, con una interpretación peyorativa, para referirse a quien labora en una organización.

Quienes aceptan el concepto "empleado", de acuerdo con Vecino (2011), consideran que el término infiere, de manera exacta, "y por tanto significa, la importancia de ser y sentirse útil en una situación determinada".Y todo, porque, en la realidad, el único bien con el que cuenta el individuo -para intercambiar en el mercado laboral- está medido por su capacidad de"emplear su fuerza o pensamiento en el logro de un resultado determinado". No obstante, dicho término entraña, en un puro sentido semiótico, una connotación de "uso", y, tal sentido, hace relación a la utilidad de sujeto, esto es, a su capacidad de producir. Ello implicaría, entonces, un desconocimiento de las aportaciones de los individuos más allá de lo tangible (la manufactura, el producto), como si se tratase de un mero objeto de consumo.

De igual manera, la disertación puede ser abordada en torno a otros términos en uso, que definen la participación de las personas en la organización. Por ejemplo, en las empresas asociadas al sector de servicios, el término de aplicación cotidiana ha sido el de "funcionario". Si bien su uso hace referencia de manera más específica a los individuos que laboran en instituciones de carácter público (DRAE, 2001), este se ha generalizado en aquellos que no son "obreros, ni trabajadores, ni empleados"; simplemente, funcionarios, que hacen que funcione la organización.

Ahora bien, dado que la dinámica de la sociedad y sus entornos (social, político, económico, cultural; entre otros) sufren manifiestas transformaciones, lo mismo sucede con las teorías de la administración. Estas han evolucionado considerando la importancia del papel de los individuos en los resultados organizacionales, conjuntamente con su rol protagónico en la nueva dinámica empresarial. Así, se ha dado paso a un concepto en el que cobra relevancia la concepción del talento humano, como el elemento que determina diferencias significativamente positivas, a la hora de evaluar los resultados de la gestión empresarial.

En tal ámbito, surge el término colaborador, que le confiere al trabajador una dimensión humana. Las nuevas corrientes de lo organizacional integran este concepto, para mostrar una asociación de los individuos a las instituciones. Más allá de lo meramente instrumental de sus capacidades físicas y mentales, entra en juego la variable de lo actitudinal, y el modelo de competencias se une al componente relacional, con la intención de considerar las personas en una dimensión holística. En otras palabras, los aportes de las personas los ven de una manera integral. Como puede observarse, es una concepción más en el sentido de la DRAE (2011), donde colaborar es "trabajar con otra u otras personas en la realización de una obra".

Tal concepto asume la actividad laboral como una concepción de asociatividad. Ya no se trata de seres individuales en función de una tarea; es un equipo, un colectivo actuando en conjunción, en una dinámica que se apropia plenamente del proceso productivo y de sus resultados, como una acción mancomunada; en fin, constituye -a la luz de las dinámicas organizacionales contemporáneas- el único modo para lograr plena productividad. En otras palabras, es una herramienta que propicia el mejoramiento del desempeño institucional. El término colaborador implica una significación social que genera empatía y un sentido de pertenencia con las organizaciones. En un sentido semántico, desecha la instrumentalización del individuo, y le otorga valor a su condición humana.

Después de establecer los conceptos de obrero, trabajador o colaborador, es necesario reflexionar acerca de las aplicaciones prácticas de dichos términos en el desarrollo y ejecución de los planes organizacionales. No nos podemos quedar reflexionando acerca de cuál concepto resulta más apropiado en términos de una significación individual o colectiva. Hay que saber que el lenguaje y su estructura semiótica (signo, significante, significado) conllevan intencionalidad; así, el término "colaborador" significaría la aceptación de una nueva dinámica, en la que prevalece la concepción holística de los individuos. Por tanto, hay que tener claro que este traduce intencionalidad y, cómo para el colectivo es 
apropiado e incide positivamente generando altos niveles de competitividad.

La discusión debe trasladarse al ámbito del desarrollo práctico del concepto, dentro de la estructura organizativa. Así, pues, resulta importante reconocer que la dinámica laboral presenta evidencias, en las que, pese a ser de uso general el empático 'colaborador', no implica un cambio del modelo organizacional tradicional jefe - empleado. De hecho, algunos jefes, gerentes y dueños de empresas continúan pensando que los trabajadores son instrumentos, y los someten, incluso, a maltratos y presiones que disienten de la consideración de los individuos, como seres humanos integrales, implícitos en la acepción del término.

Es claro que, cuando se trabaja la dimensión de'colaborador', en una estructura organizacional, hay que ir más allá de la mera consideración de lo semántico; de la simple significación. Se deben reconocer, de manera integral, todas las dimensiones de la dinámica laboral: lo contractual y lo humano. En otras palabras, la valoración de los aportes del'colaborador', en el ambiente laboral, debe ser evidente. Es frecuente encontrar empresas que solo ven los errores y pocas veces elogian los resultados positivos. Se sugiere, además de la valoración del desempeño, poner en práctica valores tradicionales de orden social, como el respeto a la dignidad del ser humano, sin distingos de ninguna naturaleza; amén de un ambiente laboral sano que anime, facilite y favorezca las sinergias requeridas para asumir el compromiso organizacional, como una responsabilidad colectiva y no individual.

En síntesis, no son las diferencias entre uno u otro término, tampoco se quiere implantar nuevos significados. Se trata de la resignificación, de asumir nuevos sentidos y comportamientos que marquen claramente la diferencia entre el antiguo y nuevo orden organizacional.

\section{Metodología}

Este estudio de caso se trabajó con el modelo de investigación cualitativa, pues se analizó un fenómeno social, en el rol laboral. Se realizó una serie de visitas a la organización objeto de estudio, enfocadas en el tema de estrés laboral. Se encontraron elementos que, indudablemente, describen el síntoma estre- sor, en su equipo humano. Lo que nos llevó a proponer una iniciativa para mejorar su ambiente laboral, a través del Departamento de Gestión Humana.

El objetivo del estudio de caso que se está describiendo es analizar, en profundidad, cómo los seres humanos desarrollan su accionar en una invariable relación con múltiples factores, enmarcados dentro de un ambiente específico. De allí, la importancia de examinar tales relaciones, para lograr una comprensión de las incidencias en su conducta. Los datos se pueden obtener, mediante el uso de entrevistas o cuestionarios; individuales o colectivos, en las que se les solicita, a los encuestados, sus impresiones en torno a vivencias y expectativas; estos, por supuesto, contienen diversos puntos de referencia. La aproximación al entorno, también, se puede explorar, estudiando documentos personales, con distintas mediciones, ya sean de orden físico, psicológico o sociológico. Es viable, también, una aproximación, mediante la indagación del entorno familiar del sujeto, así como el análisis de fuentes alternas que estén relacionadas con el caso de estudio, como las instituciones sociales y otros organismos de carácter público y/o privado.

Los estudios de casos son similares a las encuestas; con la diferencia de que presentan una mayor profundidad y una cantidad limitada de casos representativos. Es decir, se pueden considerar como más específicos. Si bien su área muestral es significativamente menor y, por tanto, su alcance está más limitado, son más profundos y sus resultados exhiben una mayor incidencia de los factores cualitativos. Su función y objetivo se fundamenta en resolver problemas puntuales; sus resultados -difícilmente replicablesdesde un punto de vista teórico, tienen pocos aportes al conocimiento científico general, pero atienden una necesidad primaria de un grupo específico, de manera más ágil y efectiva.

\section{Análisis y resultados}

\section{Test sobre estrés laboral, en la Unidad de Conductas Adictivas Psico}

Se realizó un estudio transversal acerca de indicadores de estrés a profesionales y tecnólogos de la salud. El estudio de caso exploratorio cuantitativo 
fue aplicado a los colaboradores de la empresa Psico (Unidad de Conductas Adictivas), ubicada en Cartago, Valle del Cauca, para un total 32 personas. El test incluyó todos los niveles organizacionales de la institución: jefes, profesionales, auxiliares y operarios. La caracterización muestra que la gran mayoría tiene el nivel profesional que requiere la organización. Respondieron una matriz para la identificación del nivel de estrés. Se manejó y se confirmó esta hipótesis: el nivel de atención, al igual que el género y la profesión, diferencia significativamente las respuestas de estrés. La prevalencia de síntomas de estrés estuvo representado así: moderado, en un $44 \%$ aproximadamente; y alto, en un 56\% aproximadamente. El estrés afecta, fundamentalmente, a las mujeres profesionales de atención primaria y a las tecnólogas del mismo nivel de atención.

El instrumento empleado estaba centrado en identificar la presencia de psicopatologías asociadas al estrés laboral, con lo cual se determinaría la presencia o no de agentes estresores en este ámbito laboral específico. Para ello, se analizaron las manifestaciones de la sintomatología, asociadas con tales patologías. Los resultados arrojados en la encuesta se utilizaron como insumo para conocer la fuente de los agentes estresores y, a partir de ello, establecer acciones correctivas en las áreas correspondientes. Así pues, se logró diseñar un plan de mejoras, que impactaran positivamente las diferentes dimensiones del ámbito laboral de este tipo de colaboradores.

Como se ha señalado, y de acuerdo con Peiró, (2000) la pérdida de productividad, ocasionada por el estrés, hace parte de la cotidianidad y de la sintomatología que este genera. En el ámbito laboral de la salud, no solamente los médicos están expuestos a estrés de origen asistencial. Como evidencia el trabajo de Anderson, Cooper \& Willmott (1996), esta patología está fuertemente manifiesta en otras profesiones relacionadas con la salud. Estos autores así lo evidenciaron, en un estudio hecho para determinar las fuentes de estrés; en él incluyeron, junto a médicos y a enfermeros, a otros trabajadores del sistema nacional de salud de Gran Bretaña. Por su parte, Richardsen \& Burke (1991) encontraron, en médicos canadienses, que el estrés elevado se relacionaba con una menor satisfacción en la práctica médica y con actitudes negativas hacia el cuidado de la salud de los pacientes.
El estudio de las profesiones asistenciales (sector servicios) -muy específicamente las profesiones de la salud- evidencian que la alta exigencia laboral del medio es un caldo de cultivo ideal para el surgimiento de cuadros generales de ansiedad, con alta incidencia en la manifestación de cuadros psicosomáticos. Fundamentalmente, por la alta presencia de factores de riesgo psicosocial asociados con la dinámica propia del sector (alta interacción con personas). He hecho, los profesionales de la salud han sido identificados como uno de los grupos con mayor grado de manifestación de psicopatologías asociadas con las incidencias del estrés; por esto, son uno de los grupos más abordados para el estudio de esta problemática. Pero las consecuencias van más allá: basta con observar la baja calidad del trabajo, e incluso la gran tasa de suicidios en los profesionales de este sector.

Ante tal panorama, existen varios estudios enfocados en develar la importancia de ciertos factores para la mitigación de dichos impactos. Landeweerd (1994), por ejemplo, habla de la retroalimentación, la claridad en la definición del rol y las tareas, la autonomía, las oportunidades de promoción, el liderazgo social-emocional, la edad, la experiencia laboral y el género. En cuanto al apoyo social, Baruch-Feldman, Brondolo, Ben-Dayan \& Schwartz (2002) han aportado evidencias acerca de su papel moderador del estrés.

Siguiendo con este aspecto de las relaciones sociales, vale la pena acotar un ítem más: el balance -entre esfuerzo y recompensa- parece incidir en la baja de probabilidades de ocurrencia de manifestaciones psicosomáticas asociadas con el estrés (Van Dierendonck, Schaufeli, Buunk, 2001). En buena medida, esta premisa del desbalance esfuerzo y recompensa se confirma en el modelo de estrés, propuesto por J. Siegrist (1996), aplicable no solo al estrés de las relaciones sociales, sino, en general, a la relación del hombre con el trabajo. Además, las evidencias apuntan a una mayor incidencia de los estresores en el personal femenino del sector salud. Breilh (1993) lo comprobó en un estudio acerca de los efectos del trabajo hospitalario en internos de hospitales ecuatorianos. Dicho estudio arrojó que había mayor incidencia en las mujeres $(79,3 \%)$ que en los hombres $(74,0 \%)$.

En relación con el grado de escolaridad y para el caso de los profesionales de la salud, Schaufeli (1998) ano- 
ta que, si bien entre médicos y enfermeros se refleja un nivel equiparable, en términos de agotamiento emocional, hay diferencias: los médicos tienden a una mayor despersonalización, en tanto que los enfermeros muestran un mayor grado de insatisfacción profesional.

\section{Caso Psico}

La empresa Psico es una entidad del sector de servicios, específicamente, servicios en el área de la salud mental. En ella, existen riesgos asociados, de los cuales, los más importantes son los psicosociales: los que provienen de las nuevas formas de trabajo, del dinamismo de las nuevas organizaciones y sus formas cambiantes. Son, principalmente, riesgos que provienen de las múltiples formas de atender a una clientela en interacción dinámica con el trabajador.

Como ya se explicó, se aplicó -con un criterio de inclusión específico- un test a todos los niveles organizacionales de la institución: jefes, profesionales, auxiliares y operarios; quienes, en su mayoría, tenía el nivel profesional que requiere la organización. Las encuestas se realizaron en una sesión de trabajo, para la aplicación del instrumento. La participación de los sujetos (encuestados) fue voluntaria y respondieron anónimamente los cuestionarios entregados.

Se utilizó un instrumento único: una matriz para identificar psicopatologías asociadas con el estrés, cuyas características eran: un listado de diversas psicopatologías, agrupadas en aspectos macro; un cuestionario, con una escala ordinal de 5 puntos $(0$ a 4), que expresan frecuencias crecientes acerca del modo como experimentan la sintomatología; unos valores mínimos y máximos de interpretación, 26 puntos que identificaban: ansiedad grave, nivel de estrés, entre 0 y 5 .

Tabla 1. Composición personal Psico

Composición personal Psico

\begin{tabular}{|c|c|c|c|}
\hline Género & Profesional & Tecnólogo & Total \\
\hline Mujeres & 10 & 6 & 16 \\
\hline Hombres & 10 & 6 & 16 \\
\hline
\end{tabular}

Fuente: elaboración propia mediante la Encuesta Psico (2015).
Dentro de los roles asignados, existe un sector de la parte gerencial que no tiene mucho contacto con los pacientes de conductas adictivas y de psiquiatría, pero consideran que existe sobrecarga de trabajo. Por su parte, los profesionales y técnicos, o tecnólogos, que intervienen con los pacientes, revelan altos niveles de estrés; ya que es en la intervención donde realmente se conoce la dimensión del problema. Estos profesionales utilizan técnicas de afrontamiento para superar las constantes crisis de los pacientes. En cuanto a los indicadores de presencia de estrés, 14 manifestaron sintomatología, con un nivel moderado; mientras que los 18 restantes evidencian estrés grave.

\section{Conclusiones}

Este estudio sobre el estrés nos ha permitido, a manera de reflexión, afirmar lo siguiente:

- En el mundo contemporáneo, inmerso en la dinámica globalizadora, el ámbito laboral se ha convertido en una fuente para el desgaste físi$\mathrm{co}$, al punto de considerar el estrés laboral como una epidemia entre los trabajadores de diversas ramas y profesiones. Como la gran exigencia de competitividad está consumiendo la energía física y mental de los trabajadores, son comunes las implicaciones negativas, no solo en lo personal, sino en el sentido de pertenencia institucional.

- La enorme dinámica ha traído consigo múltiples modificaciones en el proceso productivo y organizacional. Esto genera un aumento considerable en la carga de trabajo, por ende, agotamiento del personal; reducción de ganancias; deterioro de los indicadores de la percepción en seguridad laboral, propiciado por la inestabilidad en el empleo; pérdida de beneficios directos e indirectos de los trabajadores. Todo esto altera, en buena medida, el sentido mismo del laborar.

- El sector servicios, relacionado con el área de la salud, presenta altos niveles de burnout en sus trabajadores. Por tanto, es necesario plantear estrategias para su prevención y reducción. Esto también constituye un desafío, una tarea inaplazable para evitar padecimientos de orden mental que garantice la calidad asistencial del servicio.

- Entre los investigadores, es común asumir que las intervenciones enfocadas en la organización del trabajo resultan más eficaces, porque reducen las fuentes de estrés ocupacional. Sin embargo, los 
diversos y crecientes estudios en la temática, en su mayoría, están enfocados en el gerenciamiento del estrés por el individuo. En efecto, se ha constatado una escasez de estudios y análisis sobre la reducción del estrés ocupacional, aplicado, específicamente, en el sector de servicios de la salud.

- Desafortunadamente, la creencia (casi común) de las organizaciones del sector acerca de que el empleado es el responsable del estrés, lleva a pensar que las intervenciones organizacionales son de difícil implementación. Ellas temen que los programas -enfocados al mejoramiento de la calidad de vida de los colaboradores- implican un aumento de costos para la organización. Puesto que, en la práctica, ello supone un incremento en la demanda de otras reivindicaciones en pro de la reducción de la carga y jornada de trabajo.

- La calidad de un servicio está relacionada directamente con el bienestar del profesional que lo presta.

- Por tanto, son urgentes las acciones encaminadas a establecer y garantizar condiciones de trabajo digno que reduzcan el desgaste físico y emocional. Esto es esencial en el proceso organizacional del sector salud. Como también, la sensibilización de profesionales e instituciones en torno a la naturaleza misma del estrés y su ocurrencia, lo cual trae beneficios a ambos, no solo en términos de competitividad, sino también a la esencia misma del trabajo, que no es otra que procurar la construcción de una sociedad mejor para todos.

\section{Referencias}

Anderson, W.J.R., Cooper, C.L. \& Willmott, M. (1996). Sources of stress in the National Health Service: A comparison of seven occupational groups. Work and Stress, 10 (1), 88-95. http://dx. doi.org/10.1080/02678379608256787

Baruch-Feldman C., Brondolo, E., Ben-Dayan, D. \& Schwartz, J. (2002). Sources of social support and burnout, Job satisfaction, and productivity. J Occup Health Psychol, 7(1), 84-93. http://dx.doi. org/ 10.1037//1076-8998.7.1.84

Beck, U. (2008). La sociedad del riesgo mundial. En busca de la seguridad perdida. Paidós-Ibèrica (Eds.). Barcelona, España. 336 p.

Beck, U. (2006). La sociedad del riesgo. Hacia una nueva modernidad. Paidós-Ibèrica (Eds.). Barcelona, España. 400 p.
Beck, U. (2000). Un nuevo mundo feliz. La precariedad del trabajo en la era de la globalización. Paidós-Ibérica (Eds.). Barcelona, España. 344 p.

Becker, G.S. (1964). Human Capital. Princeton University Press (Eds.). N.J., USA. 22p.

Benavides, F., Delclos, J., Benach. J. \& Serra, C. (2006). Lesiones por accidentes de trabajo. Una prioridad en atención pública. Rev Esp Salud Pública, 80 (5), 553-565. http://dx.doi.org/10.1590/S113557272006000500011

Breilh, J. (1993). Trabajo hospitalario, estrés y sufrimiento mental.Salud problema, 23,21-37.http:// repositorio.uasb.edu.ec/bitstream/10644/3379/1/ Breilh\%2C\%20J-CON-097-Trabajo\%20hospitalario.pdf

Casado, J.M. (2003). El valor de la persona. Nuevos principios para la gestión del capital humano. Prentice Hall (Eds.). Madrid, España. 392 p.

Engels, F. (1972). Principios de comunismo. Ediciones Sociales (Eds.). 18p.

Gil-Monte, P. R. \& Peiró, J. M. (1997). Desgaste psíquico en el trabajo: El síndrome de quemarse. Síntesis (Eds.). Madrid, España. 144 p.

Gil-Monte, P. R., Peiró, J. M. \& Valcárcel, P. (1998). A model of burnout process development: An alternative from appraisal models of stress. Comportamento Organizacional e Gestao, 4(1), 165-179.

Henry, J. (2005). The Healthy organization. En: A.G. Antoniou \& C.L. Cooper (Eds). Research Companion to organizational Health Psychology. Cheltenham,UK. Edward Elgar. pp. 383392.

Hochschild, A. R. (1983).The managed heart: Commercialization of human feeling. University of California Press. Wiley (Eds.). Berkeley, CA., USA. 339 p.

Jiménez-Jiménez, C., \& Sánchez- Laguna, J.L. (2005). Análisis del downsizing (reducción de plantilla) como fenómeno psicosocial. Revista de Psicología del Trabajo y de las Organizaciones. 21(3), 181-206. http://www.redalyc.org/pdf/2313/231317624001. $p d f$.

Leka, S. (2004). La organización del trabajo y el estrés: estrategias sistemáticas de solución de problemas para empleadores, personal directivo y representantes sindicales. En: Protección de la salud de los trabajadores. Organización Mundial de la Salud (OMS)(Eds.). Ginebra, Suiza. 27 p. 
Landeweerd J.A. \& Boumans. (1994). NPG- the effect of work dimensions and need for autonomy on nurses' work satisfaction and health. J Occup Org Psychol, 67, 207-217

Mejía, C. (2014). Colaborador o trabajador, una disyuntiva nada inocente. http://www.sindicalistas.net/2014/02/colaborador-o-trabajador-unadisyuntiva.html.

Moreno-Jiménez, B., Garrosa, E. \& González, J.L. (2000). El desgaste profesional de enfermería: Desarrollo y validación factorial del CDPE (Professional nurses' burnout. Development and factor validation of the CDPE). Archivos prevención de riesgos laborales, 3, 18-28. https://www. uam.es/gruposinv/esalud/Articulos/Salud\%20Laboral/burnout-enfermeria-cdpe.pdf.

Narbona, K. (2010). La hipocresía de llamar a los trabajadores "colaboradores". http://wwww.elmostrador.cl/noticias/opinion/2010/12/24/la-hipocresia-de-llamar-a-los-trabajadores-colaboradores/

O.M.S. (2000). The World Health. Report 2000. Health systems: Improving performance. http://www. who.imt/whr.

OIT. Organización Internacional del Trabajo. (2010). Riesgos emergentes y nuevos modelos de prevención. Ginebra, Suiza. http://wwww.ilo.org/wcmsp5/groups/public/---ed_protect/---protrav/---safework/documents/publication/woms_124341.pdf

OIT. Organización Internacional del Trabajo. (2001). Enciclopedia de Salud y seguridad en el trabajo Tercera Edición. Sociedad Española para el estudio de la ansiedad y el estrés. Murcia, España. http://www.ucm.es/info/seas/estres_lab/enciclo/ indice_gral.htm.
Real Academia Española. (2001). Diccionario de la Lengua Española. 22ª . Ed. RAE (Eds.). Madrid, España. $h t t p: / / d l e . r a e . e s / ? w=$ diccionario

Richardsen, A.M. \& Burke, R.J. (1991). Occupational stress and job satisfaction among physicians: sex differences. Soc Sci Med, 33 (10), 1179-1187.

Rosa, C. \& Carlotto, M.S. (2005). Síndrome de Burnout e satisfação no trabalho em profissionais de una instituição hospitalar. Rev SBPH, 8(2), 1-15.

Schaufeli, W. \& Enzman, D. (1998). The Burnout companion to study \& practice: A critical analysis. Taylor \& Francis International Ltd.(Eds.). Padstow, U.K. 224 p.

Sennet, R. (2006). El respeto. Sobre la dignidad del hombre en un mundo de desigualdad. Anagrama (Eds.). Barcelona, España. 304 p.

Siegrist, J. (1996). Adverse health effects of high-effort/low-reward conditions. J Occup Health Psychol, 1(1), 27-41.

Van Dierendonck, D., Schaufeli, W.B. \& Buunk, B.P. (2001). Burnout and inequity among human service professionals: a longitudinal study. J Occup Health Psychol Jan, 6(1), 43:52.

Vecino-Pico, J. (2011). De obrero a colaborador. Una transición aún incompleta en las empresas. http://www.gestiopolis.com/obrero-a-colaborador-transicion-incompleta-empresas/

Zozaya-González, N. (2007). Las fusiones y adquisiciones como forma de crecimiento empresarial. Ministerio de Trabajo. (Eds.). Madrid, España. 33 p. http://www.ipyme.org/Publicaciones/Las\%20 fusiones $\% 20 y \% 20$ adquisiciones\%20como\%20formula\%20de\%20crecimiento\%20empresarial.pdf. 